\title{
Polêmicas sobre História Comportamental: Identificação de seus Efeitos e sua Duração
}

\section{Controversies about Behavioral History: The Identification of History Effects and their Duration}

\author{
Carlos Eduardo Costa ${ }^{*}, a$, Sérgio Dias Cirino ${ }^{b}$, Carlos Renato Xavier Cançado ${ }^{c}$, \\ $\&$ Paulo Guerra Soares ${ }^{a}$ \\ ${ }^{a}$ Universidade Estadual de Londrina, ${ }^{b}$ Universidade Federal de Minas Gerais \\ ${ }^{c}$ West Virginia University
}

\begin{abstract}
Resumo
Embora analistas do comportamento concordem que o comportamento atual seja influenciado pela história, pesquisas sobre história comportamental têm levantado várias questões polêmicas. O presente artigo analisa duas destas questões: (a) a classificação dos efeitos de história como "latentes" e (b) a possibilidade dos efeitos da história serem permanentes. Defende-se que o produto da história é um organismo modificado e não uma história armazenada. Argumenta-se que os efeitos de história são transitórios, de curta ou longa duração, em função de variáveis específicas do procedimento. $\mathrm{O}$ artigo aponta também implicações destas análises para a realização de futuros estudos na área.

Palavras-chave: História comportamental; Análise experimental do comportamento; Behaviorismo radical.

Abstract

Although behavior analysts agree that current behavior is affected by history, researches on behavior history have raised several controversial questions. The present article addresses two of these questions: (a) the classification of history effects as "latent", and (b) the possibility that history effects are permanent. It is argued that the product of history is a changed organism, not a stored history. It is suggested that history effects are transitory, short-lived or long-lasting, regarding the specific variables of the procedure. The present article also suggests implications of these analyses for future research on this area. Keywords: Behavioral history; Experimental analysis of behavior; Radical behaviorism.
\end{abstract}

O presente é o instante em que a roda do automóvel em alta velocidade toca minimamente o chão. E a parte da roda que ainda não tocou tocará num imediato que absorve o instante presente e torna-o passado. Eu, viva e tremeluzente como os instantes, acendo-me e me apago, acendo e apago, acendo e apago. (Lispector, 1973).

Abordar a questão relativa à inter-relação entre organismo e ambiente de maneira experimental permite explicitar relações de controle que poderiam não ser identificadas numa análise casual e assistemática (Skinner, 1953/1965). A descrição das variáveis das quais o comportamento é função coloca-se, portanto, como o objetivo central da Análise Experimental do Comportamento.

\footnotetext{
${ }^{*}$ Endereço para correspondência: Universidade Estadual de Londrina, Centro de Ciências Biológicas, Departamento de Psicologia Geral e Análise do Comportamento, Rodovia Celso Garcia Cid, Km 380, Caixa Postal 6001, Londrina, PR, Brasil, CEP 86051-990. E-mail: caecosta@uel.br Os autores agradecem ao professor Dr. José Antônio Damásio Abib e ao professor Dr. Carlos Eduardo Lopes, pela leitura cuidadosa e sugestões que fizeram para este artigo, mas os eximem de qualquer responsabilidade sobre seu conteúdo e eventuais equívocos. Este artigo foi parcialmente financiado pela Fundação Araucária.
}

Embora os analistas do comportamento concordem que o comportamento atual seja influenciado pela exposição prévia às contingências (e.g., Aló, 2005; Baum, 2005/ 2006; Cirino, 2001a, 2001b; Donahoe \& Palmer, 1994; Freeman \& Lattal, 1992; Morse \& Kelleher, 1977; Okouchi, 2003b; Sidman, 1960; Skinner, 1953/1965; Wanchisen, 1990; Weiner, 1964, 1983), pesquisas sobre os efeitos de contingências passadas sobre comportamento atual têm levantado várias questões, no mínimo polêmicas, para a Análise Experimental do Comportamento. O presente artigo pretende analisar duas dessas questões: (a) a classificação dos efeitos de história como "latentes" e (b) a possibilidade dos efeitos da história serem permanentes. Pretende-se também apontar implicações desta análise para a realização de futuros estudos na área.

\section{Os Efeitos da História podem ser Latentes?}

Em primeiro lugar, parece importante definir o que, neste artigo, se entende por "história comportamental". É possível encontrar diversos termos usados para se referir à exposição prévia a contingências de reforço e punição como, por exemplo, história de reforço, história de esque- 
ma, história operante, história de desempenho, história de condicionamento, história pré-experimental, efeitos de história, etc., além, é claro, de história comportamental (Cirino, 2001a, 2001b). Mas, talvez o termo história deva ser reservado para se referir a uma "exposição particular a contingências operantes ou respondentes, cuidadosamente controlada no contexto do laboratório, antes que uma 'fase teste' desejada seja introduzida" (Wanchisen, 1990, p. 32). Para Wanchisen, esta definição excluiria o que pode ser denominado de "história extra-experimental" (ou "história extralabora-tório").

Esta definição proposta por Wanchisen (1990) reduz a noção de história comportamental à história experimental, isto é, exclui qualquer estudo em que a história não tenha sido "construída" no laboratório como um estudo de história. A definição perde em abrangência, mas ganha em objetividade, o que é uma vantagem quando se trata de delinear experimentos que busquem avaliar a influência de experiências passadas sobre o comportamento atual. Deste ponto de vista, deve-se tomar cuidado quando se diz que os resultados de uma dada pesquisa foram devidos às diferentes histórias que os sujeitos (particularmente humanos) "trouxeram" para a sessão experimental. Por mais plausível - e até mesmo coerente com a forma de interpretação analítico-comportamental -, tal explicação não passaria de conjectura, pois a referida história poderia ser, no máximo, inferida, nunca observada ou manipulada. É, pois, na concepção de história expressa no texto de Wanchisen que as reflexões do presente artigo se baseiam.

Parece igualmente necessário uma definição de "efeitos de história”. De acordo com Freeman e Lattal (1992) “"efeitos de história' implicam fontes de controle sobre o comportamento presente que não foram eliminadas pelo refinamento das contingências atuais e, conseqüientemente, obscurecem as relações funcionais estabelecidas entre o responder e as contingências presentes" (p. 5). A expressão "efeito de história" é, portanto, empregada quando se observa um comportamento atual semelhante àquele que ocorreu em fases anteriores de um estudo, em condições nas quais era esperado que o comportamento se alterasse (e.g., quando as contingências de reforço são alteradas).

Um estudo que ilustra o procedimento comumente utilizado nesse tipo de pesquisa foi conduzido por Weiner (1964). Seis funcionários de um hospital foram distribuídos em dois grupos. Inicialmente (Fase de Construção da História), os participantes de um grupo foram expostos a dez sessões em FR 40 (razão fixa) ${ }^{1}$, e os participantes de outro grupo realizaram dez sessões em DRL $20 \mathrm{~s}$ (reforço diferencial de baixas taxas) ${ }^{2}$. Em uma segunda

${ }^{1} \mathrm{Em}$ um programa de reforço em FR (Fixed Ratio) um reforçador é liberado após a emissão de um número constante de respostas (Catania, 1998; Ferster \& Skinner, 1957). ${ }^{2}$ Em um programa de reforço em DRL (Differencial Reinforcement of Low rates) um reforçador é liberado se uma resposta ocorrer após a passagem de um período defi- etapa (Fase Teste), participantes de ambos os grupos foram expostos a 20 sessões em FI $10 \mathrm{~s}$ (intervalo fixo) ${ }^{3}$. Durante a Fase de Construção da História, os participantes expostos ao FR emitiram altas taxas de resposta, e os participantes expostos ao DRL emitiram baixas taxas de respostas. Na Fase Teste, participantes previamente expostos ao FR emitiram taxas de respostas relativamente mais altas do que aqueles expostos ao DRL. Isto sugere que o padrão comportamental de altas ou baixas taxas de respostas, selecionado previamente, persistiu por algum tempo após a mudança nas contingências de reforço. Estes resultados também foram replicados por Urbain, Poling, Millan e Thompson (1978) os quais utilizaram os mesmos programas de reforços com ratos. Aumento na taxa de respostas em FI após uma história em DRL foi observado no estudo de LeFrancois e Metzger (1993), no de Poling et al. com ratos e no de Weiner. Esses resultados sugerem que, apesar de um dado padrão comportamental persistir por algum tempo após a mudança nas contingências de reforço, as contingências presentes passam a controlar cada vez mais o comportamento do organismo (i.e., um novo padrão comportamental é gradualmente selecionado).

Uma questão recorrente em estudos de história comportamental diz respeito aos "efeitos latentes da história" (Tatham \& Wanchisen, 1998; Wanchisen, 1990; Wanchisen \& Tatham, 1991), referidos também como "efeitos inobserváveis da história" - inobserváveis até que condições de teste adicionais sejam arranjadas, como a administração de drogas (e.g., Barrett, 1977; Egli \& Thompson, 1989; Terrace, 1963) ou alimentação prévia (e.g., Doughty et al., 2005).

Estudos que fazem referência a efeitos latentes da história ou a efeitos inobserváveis da história baseiam-se, comumente, em achados da Farmacologia Comportamental (e.g., Barrett, 1977; Egli \& Thompson, 1989; Terrace, 1963) ${ }^{4}$. Por exemplo, Terrace (1963) estudou o efeito de drogas sobre o comportamento discriminado modelado por diferentes procedimentos (discriminação "tradicional" e discriminação "sem erros"). Pombos do grupo de discriminação "sem erros" foram condicionados a bicar um disco quando ele estivesse iluminado com a cor vermelha $\left(\mathrm{S}^{+}\right)$e não bicá-lo quando ele fosse iluminado com a cor verde $\left(\mathrm{S}^{-}\right)$. Nas sessões seguintes, padrões de linhas (vertical e horizontal) foram sobrepostos nido de tempo ( $\mathrm{t}$ ) sem que uma resposta da classe reforçada tenha sido emitida (Catania, 1998). Se uma resposta ocorrer antes do tempo especificado, o intervalo de tempo é zerado e começa a ser contado novamente; se a resposta ocorrer após o intervalo especificado, o reforço é liberado e um novo intervalo se inicia.

${ }^{3}$ Em um programa de reforço em FI (Fixed Interval) a primeira resposta emitida após um período designado de tempo é seguida por um evento reforçador (Catania, 1998; Ferster \& Skinner, 1957).

${ }^{4}$ Ressalta-se que tanto o artigo de Barrett (1977) quanto o de Terrace (1963) não fazem referência a "efeitos latentes da história". Os resultados dessas e outras pesquisas são tomados por outros autores como um exemplo de efeitos latentes da história. 
à cor do disco: linhas verticais foram sobrepostas à luz vermelha do disco e linhas horizontais foram sobrepostas à luz verde do disco. Então, ao longo de 25 tentativas as cores foram sendo esmaecidas até que somente os padrões de linhas fossem observados sobre o disco. A partir desse ponto os pombos passaram por mais 15 sessões de discriminação do padrão de linhas. Durante todo o procedimento nenhuma resposta foi emitida ante o $\mathrm{S}^{-} \mathrm{e}$ nenhum reforço foi perdido sob $\mathrm{S}^{+}$. Os pombos do outro grupo foram expostos a um treino discriminativo entre linhas horizontais $\left(\mathrm{S}^{+}\right)$e verticais $\left(\mathrm{S}^{-}\right)$sem qualquer treino progressivo como aquele descrito anteriormente. Os pombos expostos ao procedimento "tradicional" de discriminação emitiram não mais do que oito respostas ao $\mathrm{S}^{-}$nas 10 sessões que antecederam o tratamento com drogas. Portanto, os pombos dos dois grupos foram expostos a diferentes histórias de aquisição do operante discriminado, mas tinham desempenho semelhante ao final do treino de discriminação (i.e., nenhuma resposta na presença de $S^{-}$para o grupo de discriminação "sem erros" e apenas oito respostas ou menos para o grupo de discriminação "tradicional").

Durante o teste, os pombos de ambos os grupos receberam uma de quatro doses de clorpromazina, imipramina ou salina $(1 ; 3 ; 10$ e $17 \mathrm{mg})$ a cada três dias. Nem a clorpromazina nem a imipramina tiveram qualquer efeito sobre o número de respostas emitidas em face do $\mathrm{S}$, para os pombos do grupo exposto anteriormente à discriminação "sem erros"; isto é, os pombos não emitiram uma única resposta na presença do $S$. Entretanto, as duas drogas em todas as doses tiveram efeito sobre o comportamento ante o $\mathrm{S}^{-}$no grupo que aprendeu a discriminação pelo procedimento "tradicional". Os pombos deste grupo emitiram entre 5 e 3655 respostas diante do $S^{-}$nas sessões em que alguma dose das drogas foi administrada (Terrace, 1963).

Outro exemplo é o estudo de Barrett (1977) que examinou o efeito da $d$-anfetamina sobre o comportamento de macacos cujo comportamento de pressionar a barra era mantido por um programa de FI de liberação de comida e também punido pelo choque. Nesta contingência, a primeira resposta após um intervalo de 5 minutos era seguida por comida (FI $5 \mathrm{~min}$ ) e a cada $30^{\mathrm{a}}$ resposta seguia-se um choque (FR 30). Entretanto, os macacos diferiram quanto à história de aquisição do comportamento neste programa de reforço. Para os macacos de um grupo (i.e., Grupo Ingênuo), a contingência de punição em FR 30 simplesmente sobrepunha-se a um responder estável em FI 5 minutos de liberação de alimento. Os macacos de outro grupo (i.e., Grupo Experiente) foram expostos inicialmente a um programa múltiplo no qual um dos componentes era o FI 5 minutos de liberação de comida e o outro componente era de esquiva (cada resposta de pressão à barra pospunha a liberação do choque por $25 \mathrm{~s}$, que ocorria a cada $5 \mathrm{~s}$ quando a barra não era pressionada). O componente de esquiva foi poste- riormente alterado para um programa de FI 5 min de liberação de choque (i.e., a primeira resposta que ocorria após esse intervalo era seguida por um choque elétrico). O comportamento foi mantido nos dois componentes do programa múltiplo $\mathrm{FI}_{-} \mathrm{FI}^{5}$. Após um ano de exposição a esta contingência o componente de FI mantido por choque foi suspenso e um programa de reforço simples em FI 5 min com liberação de alimento foi mantido até a estabilidade do comportamento. Neste ponto, a contingência de punição em FR 30 era adicionada. Em suma, foi construída uma linha de base de comportamento punido (em FR 30) sobreposta a um programa de FI 5 minutos de liberação de alimento, contra o qual os efeitos da $d$-anfetamina foram testados. Entretanto, como ressaltado anteriormente, a história de construção dessa linha de base foi diferente para os macacos dos dois grupos. O comportamento foi igualmente suprimido quando a punição foi sobreposta ao FI para os macacos de ambos os grupos, ou seja, nenhum efeito da história foi observado nesse ponto. Todavia, a administração da $d$-anfetamina reverteu o efeito supressor da punição observado no comportamento dos macacos do Grupo Experiente, mas não daqueles do Grupo Ingênuo, o que sugere um efeito da história que não era aparente durante a linha de base.

Estudos assim conduzidos indicam diferenças no comportamento na fase de teste que podem ser atribuídas às diferentes histórias experimentais dos organismos. Esta história, por sua vez, estaria "latente", encoberta, mascarada (masked é o termo em inglês empregado por Wanchisen, 1990, p. 32) por uma condição experimental e seria trazida à tona, descoberta, pela manipulação de uma variável adicional (unmasked, Wanchisen, 1990, p. 32). Seguindo-se essa linha de argumentação, os efeitos de história poderiam ser classificados como latentes ou inobserváveis até que uma condição experimental adicional fosse arranjada para que seus efeitos pudessem ser observados no comportamento corrente.

Descrever os resultados de pesquisas, tais como aquelas apontadas acima, como evidências de um efeito latente da história comportamental pode levantar questões embaraçosas, para dizer o mínimo. Onde se encontram os efeitos da história quando nenhuma evidência deles é sugerida pela observação do comportamento atual? Dizer que os efeitos são inobserváveis ou latentes pode sugerir que variáveis existentes em outros níveis de ob-

\footnotetext{
${ }^{5}$ Em um programa múltiplo de reforço dois ou mais programas de reforço se alternam com um estímulo diferente correlacionado com cada programa de reforço (Catania, 1998; Ferster \& Skinner, 1957). Várias pesquisas demonstraram que, dada uma história específica, o comportamento de macacos pode ser mantido pela liberação de choques elétricos contingentes à resposta, assim como pela liberação de alimento, água ou outros eventos (e.g., Kelleher \& Morse, 1968; McKearney, 1968, 1969, 1972; Morse \& Kelleher, 1977). Para uma compreensão crítica desse fenômeno ver também Galbicka e Platt (1984), Laurence, Hineline e Bersh (1994) e Tatham e Wanchisen (1998).
} 
servação influenciam (ou influenciarão), de alguma forma, o comportamento. Do mesmo modo, pode dar a conotação de armazenamento, como se o organismo passasse a "conter" ou "possuir" a história comportamental.

Uma das implicações ao se falar em efeitos latentes da história é o risco de se atribuir especulativamente efeitos de história em estudos nos quais manipulações adicionais não são realizadas e os dados obtidos não podem ser suficientemente explicados pelas manipulações experimentais das contingências presentes. Esse risco deve-se ao uso de uma terminologia que favorece equívocos e não à adequação do procedimento experimental. É um problema de natureza mais conceitual do que experimental. Como se poderia afirmar que a história exerce alguma influência sobre o comportamento do organismo quando seus efeitos não são identificados a partir da observação do comportamento corrente? Nesse sentido, $\mathrm{o}$ ato de classificar efeitos de história como latentes ou inobserváveis seria quase o mesmo que atribuir resultados discrepantes de um estudo às histórias extra-experimentais dos organismos, sobre a qual o acesso e o controle podem ser inviáveis.

Como exposto anteriormente, a presente crítica referese às implicações que a terminologia empregada pode gerar. Em momento algum se questiona a qualidade do procedimento experimental dos estudos citados ou a interpretação segundo a qual certos procedimentos experimentais (e.g., administração de drogas) podem produzir diferentes efeitos em organismos com diferentes histórias (e.g., Barrett, 1977; Branch, 1991; Dews, 1955; Terrace, 1963). Pretende-se chamar a atenção para o perigo de utilizar o adjetivo "latente" para se referir a efeitos da história que não foram observados. Para isso, parece apropriado discutir, mais detalhadamente, o que se deveria entender pela expressão "efeito da história". Será traçado um paralelo entre a expressão "possuir um comportamento" e "possuir uma história" e, em seguida, será proposta uma interpretação dos "efeitos latentes da história" tomando-se por base a noção skinneriana (Skinner, 1969, 1974) de "organismo modificado" (enfatizando-se que essa modificação não pode ser tomada unicamente como modificação anátomo-fisiológica, mas deve englobar também as mudanças comportamentais).

Segundo Skinner (1974), um organismo não possui um comportamento no sentido de que o comportamento esteja no interior do organismo. "O comportamento existe somente quando está sendo executado. Sua execução requer um sistema fisiológico . . . O sistema foi modificado quando o comportamento foi adquirido, e é o sistema modificado que é 'possuído"” (Skinner, 1974, p. 151). Igualmente, dizer que o organismo "tem uma história de reforço $x$ " não significa que o organismo possui a história. $\mathrm{O}$ organismo não possui uma história assim como não possui um comportamento. A história não controla o comportamento no sentido de existir concomitantemente à exposição às contingências presentes. A história, de fato, não existe mais. Conforme Chiesa (1994), "um organismo é modificado pela exposição às contingências de reforço, e é um organismo modificado que emite respostas" (p. 30).

Os resultados de estudos, a partir dos quais efeitos de história são apontados como latentes ou inobserváveis, poderiam ser (re)interpretados como se segue: dois organismos são diferentemente modificados pela exposição prévia a diferentes contingências (por exemplo, um deles responde em FR e outro em DRL). Quando expostos a uma nova condição experimental (por exemplo, se ambos respondem em FI), os dois organismos são novamente modificados. Se expostos por tempo suficiente a esta nova contingência, o comportamento de ambos pode ser suficientemente semelhante a ponto de se dizer que o comportamento presente está sob controle da contingência presente e que a história não exerce mais influência sobre o comportamento em questão (cf. Cole, 2001). Seria possível dizer que não há mais efeitos da história no comportamento atual desses organismos. Nesse ponto do estudo, uma alteração na contingência (por exemplo, a administração de uma droga ou mesmo a manipulação de outras variáveis) pode produzir alterações diferentes no comportamento de cada um dos organismos. Entretanto, isso ocorre não porque os efeitos da história estivessem latentes, mas porque os organismos que respondem de maneira semelhante em FI não são organismos idênticos (i.e., não se tornaram idênticos porque agora se comportam de maneira semelhante em FI). Ambos foram diferencialmente modificados pela exposição a contingências diversas (de FR e DRL em nosso caso hipotético). As contingências históricas são responsáveis pelos diferentes padrões comportamentais observados na última manipulação. Parece desnecessário - e mecanicista - supor que os efeitos permaneceram latentes e que esses efeitos latentes foram os responsáveis pela mudança posterior no comportamento sob novas contingências. Apesar de emitirem um padrão comportamental semelhante em FI, isso não significa que ambos serão afetados da mesma maneira por uma mesma manipulação experimental subseqüente. A esse respeito Skinner (1969) afirmou:

O sistema complexo que nós chamamos de organismo tem uma história elaborada e amplamente desconhecida que o dota de certa individualidade. Dois organismos não entram em um experimento precisamente nas mesmas condições e nem são afetados do mesmo modo pelas contingências no experimento. (É característica da maioria das contingências que elas não sejam controladas precisamente e, de qualquer forma, elas só são efetivas em combinação com o comportamento que o organismo traz para o experimento) (p. 111-112).

É preciso um parêntese importante neste ponto. Quando se diz que um indivíduo exposto a determinadas contingências torna-se um "organismo modificado", o 
organismo em questão não deveria ser entendido apenas como um "organismo biologicamente modificado". É mais do que isso. Embora o behaviorismo skinneriano tome em consideração o organismo biológico, ele vai além na delimitação do seu objeto.

$\mathrm{O}$ behaviorismo radical é uma filosofia do corpo. $\mathrm{O}$ corpo é máquina bioquímica e ação. $\mathrm{O}$ corpo como máquina bioquímica ou estrutura - ou ainda, o corpo-com-cérebro - é objeto de estudo da física, química, fisiologia e neurociência. O corpo como ação ou comportamento é o objeto de estudo das ciências da variação e seleção: a etologia, a análise do comportamento e uma parte da antropologia. Como filosofia do corpo, o behaviorismo radical defende um conceito de corpo que denuncia as insuficiências do materialismo e do mecanicismo. O materialismo e a teoria do homem como autômato só encontram refúgio no corpo como máquina bioquímica. Como filosofia do corpo, o behaviorismo radical sugere como é possível recuperar o conceito de corpo sem reintroduzir sub-repticiamente a doutrina metafísica do materialismo (Abib, 2001, p. 24).

Skinner (1989/1991) diferencia organismo de pessoa, enfatizando a diferença entre a história filogenética (que dá origem ao organismo propriamente dito, o executor) e a história ontogenética (que dá origem à pessoa). Uma história (ontogenética) de reforço, como aquelas descritas nos exemplos do presente artigo, produz uma nova "pessoa" ou um "organismo modificado" - tomando-se uma acepção mais ampla de "organismo", isto é, um organismo que engloba aqui um sistema biológico complexo com diferentes probabilidades de se comportar dessa ou daquela maneira sob certas circunstâncias. Nas palavras de Skinner (1974):

$\mathrm{O}$ ambiente fez sua primeira grande contribuição durante a evolução das espécies, mas ele exerce um tipo diferente de efeito durante a vida do indivíduo, e a combinação dos dois efeitos é o comportamento que observamos em qualquer dado momento (p. 19).

$\mathrm{Se}$, de acordo com a tese do organismo modificado, o "organismo" for tomado apenas como um sistema biológico e, por conseguinte, a modificação for entendida apenas como a modificação neste sistema biológico (e não englobar as modificações nas probabilidades do comportamento) então teremos uma tese que pende para o reducionismo biológico e também para o mecanicismo - visto que a modificação no organismo biológico serviria, em certa medida, para "preencher a lacuna" entre aspectos do ambiente e a resposta. A separação de organismo biológico de um lado e o chamado repertório comportamental de outro - assim como a separação entre organismo e ambiente é, quando muito, didática. A teoria conseqüencialista skinneriana é relacional (Abib, 2004) e, portanto, deste ponto de vista, não há comportamento sem organismo nem organismo sem comportamento.
Voltando à questão central, a saber, "os efeitos da história podem ser latentes?", a resposta parece ser não. Se alguém procura pelos efeitos que a história exerce sobre o comportamento de um dado organismo, e se o comportamento em andamento não sugere nenhuma diferença em razão da história, então não parece haver vantagens em supor que o efeito exista, mas esteja latente. Não se pode igualmente afirmar, de uma perspectiva analíticocomportamental, que o efeito está no organismo, como sistema biológico. Desta perspectiva, não seria possível encontrar traços biológicos que, sozinhos, remontem à história. $\mathrm{O}$ produto da história é um sistema complexo modificado - que envolve um organismo biológico $e$ diferentes probabilidades para a ação - e não uma história armazenada. Portanto, a modificação do sistema biológico assim como das probabilidades para a ação (economicamente denominados no presente artigo pela expressão "organismo modificado") são produtos das contingências de reforço e nenhum deles pode ser tomado como explicação para o comportamento presente. O uso do termo "latente" pode favorecer a metáfora do armazenamento - presente em concepções tradicionais de percepção e memória ou mesmo em concepções analíticocomportamentais mal compreendidas, tais como repertório ou história comportamental - incansavelmente criticada por Skinner (1974, 1981/1988, 1989), além de sugerir que são esses "efeitos latentes" as causas do comportamento em outro dado momento.

Por outro lado, seria sensato supor que organismos com histórias diferentes, mesmo quando se comportam de forma semelhante em uma situação presente, podem ser diferentemente afetados por uma mesma variação no procedimento? Os resultados experimentais sugerem que sim (e.g., Barrett, 1977; Terrace, 1963). Mas dizer que histórias diferentes produzem organismos diferentes, e que estas diferenças serão observadas somente se características idiossincráticas do ambiente forem modificadas, não é o mesmo que dizer que o efeito da história esteja latente. Histórias ontogenéticas específicas modificam a probabilidade dos organismos se comportarem de determinadas maneiras sob certas circunstâncias, mesmo quando, em outras circunstâncias, estes organismos comportam-se de forma semelhante. A noção de uma história latente aumenta a probabilidade de uma concepção equivocada de que dado o "gatilho" específico o comportamento infalivelmente surgirá. A concepção de que o organismo como um todo é modificado - em interação com as contingências presentes - sugere que experiências passadas podem afetar o comportamento atual, mas o faz de um modo mais abrangente, mais dinâmico. A história, como defendida aqui, é parte do processo que chamamos comportamento, ela não é uma "coisa" que os organismos possuem.

Em última instância, as causas do comportamento estão nas contingências históricas filogenéticas e ontogenéticas - incluindo-se as contingências culturais (Andery 
\& Sério, 2001; Chiesa, 1994; Micheletto, 1997a, 1997b; Skinner, 1953/1965, 1974, 1981/1988, 1989/1991). De um ponto de vista analítico-comportamental não precisamos trazer as causas do comportamento para o ambiente imediato - quer na forma de um efeito latente, quer na forma de um traço biológico no organismo. Talvez seja possível falar de um organismo (como um todo) modificado e essa concepção parece ter a vantagem de voltar a observação para as contingências de reforço que "produziram" o indivíduo que se comporta. O ponto crítico é a compreensão das contingências que são responsáveis pelos efeitos atuais - contingências do ambiente imediato e da história do organismo.

\section{Os Efeitos da História podem ser Permanentes?}

Outra questão controversa em relação à história comportamental foi apontada por Wanchisen (1990) e desenvolvida posteriormente (Tatham \& Wanchisen, 1998; Wanchisen \& Tatham, 1991): os efeitos de história são permanentes ou transitórios? Para Sidman (1960) os efeitos da história comportamental são aqueles que ocorrem num estado de transição entre duas contingências de reforço. Em outros termos, o objeto de estudo do analista de comportamento que estuda experimentalmente os efeitos da história é o comportamento que ocorre no intervalo entre um estado estável e, supostamente, outro estado estável. Segundo este ponto de vista (e.g., Baron \& Leinenweber, 1995; Cole, 2001; Freeman \& Lattal, 1992; Okouchi, 2003a, 2003b), os efeitos da história comportamental são sempre transitórios (i.e., eles estão no interstício de dois estados estáveis). Entretanto, outros autores (e.g., Tatham \& Wanchisen, 1998; Wanchisen \& Tatham, 1991) sugeriram que, em alguns casos, os efeitos da história poderiam ser considerados permanentes.

Tatham e Wanchisen (1998) defenderam a possibilidade de efeitos permanentes da história comportamental citando os resultados de Wanchisen, Sutphin, Balogh e Tatham (1998) que estudaram o efeito da exposição prévia a um programa de reforço em FR sobre a exposição subseqüente a um programa em DRL e vice-versa. Trinta e oito ratos foram distribuídos em três grupos. Os ratos do Grupo DRL foram expostos aos seguintes programas de reforço, nesta ordem: DRL, FR e DRL. Os ratos do Grupo FR-I foram expostos aos seguintes programas de reforço: FR e DRL. Os ratos do Grupo FR-II foram expostos a um programa de FR e, então, passaram por um período sem exposição a qualquer programa de reforço e foram novamente expostos a outro período em FR. O Grupo DRL emitiu taxas de respostas mais baixas do que o Grupo FR-I quando ambos estiveram respondendo em um programa de FR, sugerindo assim um efeito entre grupos. A taxa média de respostas do Grupo FRI foi mais alta na primeira exposição ao FR do que na segunda exposição (i.e., após uma história interveniente de DRL) o que sugere também um efeito intragrupo. Os autores relataram que fizeram diversas manipulações (e.g., variando os parâmetros do DRL e do FR, incluindo sinalizações das mudanças na contingência e uso de limited hold) para tentar aumentar a taxa de respostas e diminuir a pausa pós-reforço dos ratos expostos ao programa de FR após uma história de DRL. Estas manipulações foram tentadas por mais de um ano e nenhuma foi capaz de alterar o efeito que a história de exposição a um programa de DRL teve sobre o comportamento subseqüente em FR. Com isso, os autores sugeriram que o efeito da exposição a um programa de reforço em DRL produziu mudanças no comportamento em um programa de reforço em FR subseqüente e que tais mudanças foram difíceis de serem revertidas.

Interessado nesta questão Cole (2001) realizou um estudo com o objetivo de avaliar, entre outros aspectos, a questão da transitoriedade ou permanência dos efeitos de história. Ratos experimentalmente ingênuos foram distribuídos em cinco condições. Grupo I: FI; Grupo II: DRL e FI; Grupo III: FR e FI; Grupo IV: FR, DRL e FI e Grupo V: DRL, FR e FI. A exposição a qualquer um dos programas de reforço, inclusive ao programa de FI, durou 80 sessões no mínimo. Em todas as condições, o comportamento dos ratos ficou gradativamente sob controle da contingência de FI, isto é, os efeitos de história foram considerados transitórios. O efeito mais visível da história foi observado no comportamento dos ratos do Grupo V durante a exposição ao FR. Os ratos deste grupo emitiram taxa de respostas sob FR mais baixa do que aqueles que foram expostos ao programa de FR logo no início do experimento (Grupos III e IV). Esse resultado, o de que uma exposição anterior a uma contingência de DRL diminui a taxa de respostas quando os ratos são expostos a um programa de FR subseqüentemente, está em concordância com aqueles apresentados por Wanchisen et al. (1998).

Segundo observações de Cole (2001), os estudos que relataram dados indicadores de que os efeitos da história poderiam ser irreversíveis (e.g., LeFrancois \& Metzger, 1993; Urbain et al., 1978 - se considerados os resultados até a fase de introdução da droga - e Wanchisen, Tatham, $\&$ Mooney, 1989) realizaram de 15 a 40 sessões na fase de teste (i.e., durante a exposição ao FI) ${ }^{6}$ e estudos que relataram efeitos transitórios de curta ou longa duração (e.g., Freeman \& Lattal, 1992) realizaram de 60 a 90 sessões. Cole (2001) apontou para o fato de que após 80 a 100 sessões de exposição ao FI seus resultados estavam mais de acordo com os estudos que relataram efeitos transitórios. Porém, tomando-se os dados apenas das primeiras 40 sessões em FI, seus resultados eram semelhantes àqueles oferecidos pelos autores que relataram efeitos da história ao final do experimento. Cole concluiu que os

${ }^{6}$ Isto é, os efeitos não foram revertidos quando da conclusão dos experimentos. Entretanto, em nenhum destes estudos os autores defenderam abertamente que os efeitos da história pudessem ser permanentes. 
resultados de sua pesquisa davam suporte para a generalização de que expor os organismos a uma longa exposição sob um programa de reforço em FI poderia dissipar os efeitos da história de reforço. Apesar disso, o próprio autor reconheceu que o efeito da exposição prévia ao DRL sobre o comportamento subseqüente em FR não foi um efeito que desapareceu até o final do estudo e sugeriu que pesquisas adicionais fossem realizadas para tornar possível entender melhor estes efeitos.

Uma interpretação alternativa seria que os efeitos de história podem ser transitórios, de curta ou longa duração (ou mais ou menos persistentes), em decorrência de variáveis específicas do procedimento. Uma linha de investigação interessante acerca da história comportamental seria especificar as variáveis experimentais que produzem maior ou menor persistência comportamental. Conforme sugeriram Lattal e Neef (1996), “a duração de uma história poderia afetar a sua persistência” (p. 216). Quanto mais extensa fosse a fase de construção da história maior poderia ser a fase de transição quando as contingências mudassem. A maioria dos estudos sobre os efeitos da história mantém a fase de construção da história até que um comportamento estável seja estabelecido. A série de experimentos conduzidos por Weiner (1982), com pacientes psiquiátricos e não-psiquiátricos, é uma exceção. No estudo de Weiner, tanto uma história de 16 sessões de DRO $10 \mathrm{~s}$ (reforço diferencial de outras respostas) ${ }^{7}$ quanto uma história de oito sessões, neste mesmo programa de reforço, reduziram a taxa de respostas em um programa de FR subseqüente, quando comparado com o comportamento em FR de pessoas sem história experimental de DRO. Ou seja, diferentes durações da fase de construção da história (8 ou 16 sessões de DRO) tiveram o mesmo efeito no comportamento subseqüente em FR. Apesar desse resultado responder negativamente à sugestão de Lattal e Neef (1996), outras pesquisas necessitam ser realizadas para responder se a extensão da história pode afetar a persistência comportamental quando as contingências de reforço são alteradas. Segundo Freeman e Lattal (1992), o que importa em estudos de história não é o fato de que seus efeitos diminuem com o tempo, mas que eles persistem por um tempo e que a análise das determinantes dessa persistência seja realizada.

Em outras palavras, os efeitos da história parecem ser sempre transitórios, mas em algumas ocasiões essa transição é mais rápida do que em outras, e uma linha de pesquisa importante em história comportamental é determinar as variáveis que contribuem para o aumento ou diminuição dessa fase de transição (ou da "persistência comportamental"). Uma linha de pesquisa que aparentemente trabalha precisamente com essa questão é a do

\footnotetext{
${ }^{7}$ Em um programa de reforço em DRO (Diffencial Reinforcement of Other behavior) um reforçador é liberado se o organismo NÃO emitir uma resposta específica (e.g., pressionar um botão) durante um determinado período de tempo. Caso a resposta específica seja emitida o intervalo é reiniciado (Catania, 1998).
}

momento comportamental ou resistência à mudança (e.g., Lattal, 1989; Nevin, 1974; 1988; Nevin, Mandell, \& Atak, 1983). Seria interessante que pesquisadores interessados em ambas as áreas, história comportamental e momento comportamental, desenvolvessem projetos conjuntos, em que tais questões possam ser sistematicamente avaliadas ${ }^{8}$.

\section{Considerações Finais}

Estudos sobre efeitos da história têm produzido evidências sistemáticas acerca da importância da história na determinação do comportamento dos organismos. Entretanto, tais estudos têm levantado várias questões, de cunho tanto teórico-conceitual quanto metodológico. O presente trabalho discutiu duas questões: (a) a identificação dos efeitos da história - especificamente o conceito de "efeitos latentes da história" e como as experiências prévias podem exercer influência sobre o comportamento presente - e (b) a duração dos efeitos da história.

Essas duas questões parecem interligadas. No presente trabalho defendeu-se que a história modifica o sistema complexo a que se chama organismo - que inclui, além do sistema biológico, as diferentes probabilidades de se comportar de certas maneiras sob certas condições - e que é um organismo modificado que interage com seu ambiente imediato (portanto, a história não está latente ou armazenada). Se o produto da história é um sistema complexo modificado (e em modificação), este organismo provavelmente nunca mais voltará a se comportar exatamente da mesma maneira no futuro. Evidências que parecem sustentar esta afirmação são, por exemplo, a observação de que uma "volta" do comportamento ao nível operante dificilmente é atingida, mesmo após muitas horas de extinção (cf. Millenson, 1967/1975) e a observação de que a "reaquisição" do comportamento de pressionar a barra após a extinção é mais rápida do que a aquisição inicial desse comportamento (cf. Catania, 1998; Sidman, 1960). Adicionalmente, resultados sugerem que em uma seqüência de exposição FR-FI-FR-FI, o comportamento exibido na segunda exposição ao FI difere, em alguns aspectos, do comportamento exibido na primeira exposição ao FI (e.g., Baron \& Leinenweber, 1995; Wanchisen et al., 1989).

Podemos supor, então, que as mudanças produzidas pela história de reforço serão permanentes? Se por "mudança permanente" se quer dizer que o comportamento do organismo será sempre e em qualquer momento o produto exclusivo da história, a resposta parece ser não. O comportamento é função da relação de um organismo como um todo (produto, entre outras, de sua história ontogenética) com seu ambiente imediato. $\mathrm{O}$

\footnotetext{
${ }^{8}$ A inter-relação entre as áreas de momento comportamental, sensibilidade comportamental e efeitos da história foi exposta e discutida por Santos (2005)
} 
comportamento não é função simplesmente da história comportamental ou do ambiente presente, mas de ambos. Portanto, dadas as características do ambiente imediato, uma história ontogenética particular pode ser irrelevante. A questão que parece importante, nesse caso, não é saber se os efeitos daquela história estão no organismo (seja em uma estrutura cognitiva hipotética seja no organismo biológico em si). A questão importante de um ponto de vista analítico-comportamental - parece ser identificar quais contingências (históricas) aumentam a probabilidade de ocorrência de um dado comportamento no futuro, apesar de não alterar a probabilidade do comportamento em uma contingência subseqüente mais imediata.

Enfim, a história "está presente" apenas no sentido de que há um organismo modificado que exibe um padrão comportamental que foi selecionado ao longo de sua ontogênese e é sobre este "material" que as contingências de reforço correntes exercerão seu efeito.

Por outro lado, se por "mudança permanente" se quer dizer que um organismo foi modificado por sua história e que esta modificação poderá - sob certas circunstâncias - fazer diferença na interação desse organismo com seu ambiente imediato, a resposta poderia ser sim: os efeitos da história podem ser permanentes (i.e., podem alterar a probabilidade do comportamento do organismo em um período posterior que transcende as contingências em vigor no presente) e, talvez por isso, seja prudente tê-la em consideração, mesmo quando ela parece não ser importante em um dado momento. Todavia, não se pode perder de vista que o aspecto mais fundamental dessa análise é que devemos tratar dos efeitos da história quando eles são identificados no comportamento corrente. Porém, dizer que o produto da história é um organismo permanentemente modificado não equivale a dizer que o efeito da história será observado indefinidamente no comportamento estudado (como sugerem os defensores do efeito permanente da história, apontados anteriormente). Os efeitos durarão enquanto as contingências o permitirem.

Também se deve enfatizar que a resposta afirmativa a essa questão não pode ser compreendida como uma sugestão de que os efeitos da história podem, então, ficar latentes até serem "revelados" pela contingência presente. A tese do "organismo modificado" não sustenta ou não suporta a tese do "efeito latente da história". Como já defendido em outros pontos do presente artigo, a tese do "efeito latente da história" conduz a equívocos conceituais e a proposições que são incompatíveis com a filosofia do behaviorismo radical. A tese do "efeito latente da história" recende a uma filosofia mecanicista da interação do homem com o mundo.

Tais questões são polêmicas e complexas, e futuras reflexões se fazem necessárias, bem como abordar outras questões relacionadas, tais como: quais seriam os critérios necessários para que um experimento possa ser clas- sificado como um estudo sobre história comportamental? (ver, por exemplo, Tatham \& Wanchisen, 1998). E uma questão diretamente relacionada a essa: seria a "história comportamental" uma área de estudo independente dentro da Análise do Comportamento? Não seriam todos os estudos em Análise do Comportamento estudos sobre história comportamental?

\section{Referências}

Abib, J. A. D. (2001). Arqueologia do behaviorismo radical e o conceito de mente. In H. J. Guilhardi, M. B. B. P. Madi, P. B. P. S. Queiroz. \& M. C. Scoz (Eds.), Sobre comportamento e cognição: Expondo a variabilidade (Vol. 7, pp. 20-35) Santo André, SP: ESETec.

Abib, J. A. D. (2004). O que é comportamentalismo? In M. Z. S. Brandão, F. C. S. Conte, F. S. Brandão, Y. K. Ingberman, V. L. M. da Silva, \& S. M. Oliane (Eds.), Sobre comportamento e cognição: Contingências e metacontingências: Contextos socioverbais e o comportamento do terapeuta (Vol. 13, pp. 52-61). Santo André, SP: ESETec.

Aló, R. M. (2005). História de reforçamento. In J. AbreuRodrigues \& M. R. Ribeiro (Eds.), Análise do comportamento: Pesquisa, teoria e aplicação (pp. 45-62). Porto Alegre, RS: Artmed.

Andery, M. A. P. A., \& Sério, T. M. A. P. (2001). Behaviorismo radical e os determinantes do comportamento. In $\mathrm{H}$. J. Guilhardi, M. B. B. P. Madi, P. B. P. S. Queiroz, \& M. C. Scoz (Eds.), Sobre comportamento e cognição: Expondo a variabilidade (Vol. 7, pp. 159-163). Santo André, SP ESETec.

Baron, A., \& Leinenweber, A. (1995). Effects of a variableratio conditioning history on sensitivity to fixed-interval contingencies in rats. Journal of the Experimental Analysis of Behavior, 63, 97-110.

Barrett, J. E. (1977). Behavioral history as a determinant of effects of d-amphetamine on punished behavior. Science, 198, 67-69.

Baum, W. M. (2006). Compreender o behaviorismo: Comportamento, cultura e evolução (M. T. A. Silva, M. A. Matos, G. Y. Tomanari, E. Z. Tourinho, \& F. Dentello, Trads., 2. ed.). Porto Alegre, RS: Artes Médicas. (Original publicado em 2005)

Branch, M. N. (1991). Behavioral pharmacology. In I. H. Iversen \& K. A. Lattal (Eds.), Experimental analysis of behavior, part 2 (pp. 21-77). New York: Elsevier Science.

Catania, A. C. (1998). Learning (4nd ed.). Trenton, NJ: Prentice Hall.

Chiesa, M. (1994) Radical behaviorism: The philosophy and the science. Boston: Authors Cooperative.

Cirino, S. (2001a). Detecção da história de reforçamento: Problemas metodológicos para lidar com a história passada. In H. J. Guilhardi, M. B. B. P. Madi, P. P. Queiroz, \& M. C. Scoz (Eds.), Sobre comportamento e cognição: Expondo a variabilidade (Vol. 8, pp. 137-147). Santo André, SP: ESETec.

Cirino, S. D. (2001b). O que é história comportamental. In H. J. Guilhardi, M. B. B. P. Madi, P. B. P. S. Queiroz, \& M. C. Scoz (Eds.), Sobre comportamento e cognição: Expondo a variabilidade (Vol. 7, pp. 153-158). Santo André, SP: ESETec. 
Cole, M. R. (2001). The long term effect of high and low rate responding histories on fixed-interval responding in rats. Journal of the Experimental Analysis of Behavior, $75,43-54$

Dews, P. B. (1955). Studies on behavior. I. Differential sensitivity to pentobarbital of pecking performance in pigeons depending on the schedule of reward. Journal de Pharmacologie, 113, 393-401.

Donahoe, J. W., \& Palmer, D. C. (1994). Learning and complex behavior. Needham Heights, MA: Allyn and Bacon.

Doughty, A. H., Cirino, S., Mayfield, K. H., Da Silva, S. P., Okouchi, H., \& Lattal, K. A. (2005). Effects of behavioral history on resistance to change. Psychological Record, 55, 315-330.

Egli, M., \& Thompson, T. (1989). Effects of methadone on alternative fixed-ratio fixed-interval performance: Latent influences on schedule-controlled responding. Journal of the Experimental Analysis of Behavior, 52(2), 141-153.

Ferster, C. B., \& Skinner, B. F. (1957). Schedules of reinforcement. New York: Appleton Century Crofts.

Freeman, T. J., \& Lattal, K. A. (1992). Stimulus control of behavior history. Journal of the Experimental Analysis of Behavior, 57, 5-15.

Galbicka, G., \& Platt, J. R. (1984). Interresponse-time punishment: A basis for shock-maintained behavior. Journal of the Experimental Analysis of Behavior, 41(3), 291-308.

Kelleher, R. T., \& Morse, W. H. (1968). Schedules using noxious stimuli: III. Responding maintained with responseproduced electric shocks. Journal of the Experimental Analysis of Behavior, 11(6), 819-838.

Lattal, K. A. (1989). Contingencies on response rate and resistance to change. Learning and Motivation, 20, 191-203.

Lattal, K. A., \& Neef, N. A. (1996). Recent reinforcementschedule research and applied behavior analysis. Journal of Applied Behavior Analysis, 29(2), 213-230.

Laurence, M. T., Hineline, P. N., \& Bersh, P. J. (1994). The puzzle of responding maintained by response-contingent shock. Journal of the Experimental Analysis of Behavior, 61(2), 135-153.

LeFrancois, J. R., \& Metzger, B. (1993). Low-response-rates conditioning history and fixed-interval responding in rats. Journal of the Experimental Analysis of Behavior, 59, 543 549.

Lispector, C. (1973). Água viva (2. ed.), Rio de Janeiro, RJ: Artenova.

McKearney, J. W. (1968). Maintence of responding under a fixed-interval schedule of eletric shock-presentation. Science, 160, 1249-1251.

McKearney, J. W. (1969). Fixed-interval schedules of electric shock presentation: Extinction and recovery of performance under different shock intensities and fixed-interval durations. Journal of the Experimental Analysis of Behavior, 12(2), 301-313.

McKearney, J. W. (1972). Maintenance and suppression of responding under schedules of electric shock presentation. Journal of the Experimental Analysis of Behavior, 17(3), 425-432.

Micheletto, N. (1997a). Bases filosóficas do behaviorismo radical. In R. A. Banaco (Ed.), Sobre comportamento e cognição: Aspectos teóricos, metodológicos e de formação em análise do comportamento e terapia cognitivista (Vol. 1, pp. 29-44). Santo André, SP: ARBytes.
Micheletto, N. (1997b). Variação e seleção: As novas possibilidades de compreensão do comportamento humano. In R. A. Banaco (Ed.), Sobre comportamento e cognição: Aspectos teóricos, metodológicos e de formação em análise do comportamento e terapia cognitivista (Vol. 1, pp. 117-131). Santo André, SP: ARBytes.

Millenson, J. R. (1975). Princípios de análise do comportamento (A. A. Souza \& D. Rezende, Trads.). Brasília, DF: Coordenada. (Original publicado em 1967)

Morse, W. H., \& Kelleher, R. T. (1977). Determinants of reinforcement and punishment. In W. K. Honig, \& J. E. R. Staddon (Eds.), Handbook of operant behavior (pp. 174200). Englewood Cliffs, NJ: Prentice-Hall.

Nevin, J. A. (1974). Response strength in multiple schedules. Journal of the Experimental Analysis of Behavior, 21(3), 389-408.

Nevin, J. A. (1988). Behavioral momentum and the partial reinforcement effect. Psychological Bulletin, 103(1), 44-56.

Nevin, J. A., Mandell, C., \& Atak, J. R. (1983). The analysis of behavioral momentum. Journal of the Experimental Analysis of Behavior, 39(1), 49-59.

Okouchi, H. (2003a). Effects of differences in interreinforcer intervals between past and current schedules on fixedinterval responding. Journal of the Experimental Analysis of Behavior, 79, 49-64.

Okouchi, H. (2003b). Stimulus generalization of behavioral history. Journal of the Experimental Analysis of Behavior, 80, 173-186.

Santos, C. V. (2005). Momento comportamental. In J. AbreuRodrigues \& M. R. Ribeiro (Eds.), Análise do comportamento: Pesquisa, teoria e aplicação (pp. 63-80). Porto Alegre, RS: Artmed.

Sidman, M. (1960). Tactics of scientific research. New York: Basic Books.

Skinner, B. F (1965). Science and human behavior. New York: The Free Press. (Original publicado em 1953)

Skinner, B. F. (1969). Contingencies of reinforcement. New York: Appleton-Century-Crofts.

Skinner, B. F. (1974). About behaviorism. New York: Vintage Books.

Skinner, B. F. (1988). Selection by consequences. In A. C. Catania \& S. Harnad (Eds.), The selection of behavior: The operant behaviorism of B. F. Skinner: Comments and consequences (pp. 11-76). New York: Cambridge University Press. (Original publicado em 1981)

Skinner, B. F. (1989). The origins of cognitive thought. American Psychologist, 44(1), 13-18.

Skinner, B. F. (1991). Questões recentes na análise comportamental (A. L. Neri, Trad.). Campinas, SP: Papirus. (Original publicado em 1989)

Tatham, T. A., \& Wanchisen, B. (1998). Behavioral history: A definition and some common findings from two areas of research. Behavior Analyst, 21, 241-251.

Terrace, H. S. (1963). Errorless discrimination learning in the pigeon: Effects of chlorpromazine and imipramine. Science, 140, 318-319.

Urbain, C., Poling, A., Millan, J., \& Thompson, T. (1978). dAmphetamine and fixed-interval performance: Effects of operant history. Journal of the Experimental Analysis of Behavior, 29, 385-392.

Wanchisen, B. A. (1990). Forgetting the lessons of history. Behavior Analyst, 13, 31-37. 
Wanchisen, B. A., Sutphin, G. E., Balogh, S. A., \& Tatham, T. A. (1998). Lasting efects of a behavioral history of low-rate responding in rats. Learning and Motivation, 29, 220-235.

Wanchisen, B. A., \& Tatham, T. A. (1991). Behavioral history: A promising challenge in explaining and controlling human operant behavior. Behavior Analyst, 14,139-144.

Wanchisen, B. A., Tatham, T. A., \& Mooney, S. E. (1989). Variable-ratio conditioning history produces high- and lowrate performances in rats. Journal of the Experimental Analysis of Behavior, 52, 167-179.

Weiner, H. (1964). Conditioning story and human fixed-interval performance. Journal of the Experimental Analysis of Behavior, 7, 383-385.

Weiner, H. (1982). Histories of response omission and human operant behavior under a fixed-ratio schedule of reinforcement. Psychological Record, 32(3), 409-434.

Weiner, H. (1983). Some thoughts on discrepant human-animal performances under schedules of reinforcement. Psychological Record, 33(4), 521-532. 